\title{
Oysters and Vibrios as a Model for Disease Dynamics in Wild Animals
}

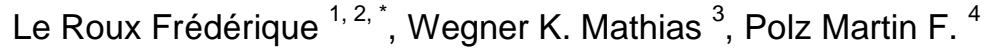

${ }^{1}$ IFREMER, Unite Physiol Fonct Organismes Marins, ZI Pointe Diable, CS 10070, F-29280 Plouzane, France.

2 Univ Paris 06, Univ Paris 04, CNRS, UMR 8227, Integrat Biol Marine Models,Stn Biol Ros, CS 90074, F-29688 Roscoff, France.

${ }^{3}$ Helmholtz Zentrum Polar \& Meeresforsch, AWI, Coastal Ecol, Waddensea Stn Sylt, Hafenstr 43, D25992 List Auf Sylt, Germany.

${ }^{4}$ MIT, Parsons Lab Environm Sci \& Engn, 77 Massachusetts Ave, Cambridge, MA 02139 USA.

* Corresponding author : Frédérique Le Roux, email address : frederique.le-roux@sb-roscoff.fr

\begin{abstract}
:
Disease dynamics in the wild are influenced by a number of ecological and evolutionary factors not addressed by traditional laboratory-based characterization of pathogens. Here we propose the oyster, Crassostrea gigas, as a model for studying the interaction of the environment, bacterial pathogens, and the host in disease dynamics. We show that an important first step is to ask whether the functional unit of pathogenesis is a bacterial clone, a population, or a consortium in order to assess triggers of disease outbreaks and devise appropriate monitoring tools. Moreover, the development of specific-pathogenfree (SPF) oysters has enabled assessment of the infection process under natural conditions. Finally, recent results show the importance of microbial interactions and host genetics in determining oyster health and disease.
\end{abstract}

\section{Trends}

Knowledge of the genetic structure of the Vibrio population provides a framework for mapping disease properties and the analysis of the evolutionary and ecological dynamics of these organisms.

Specific-pathogen-free (SPF) oysters have recently been developed, enabling the study of disease ecology in ocean environments.

High-resolution environmental sampling, population and functional genomics, combined with highthroughput infection assays, can lead to identification of the functional unit of pathogenesis in oysters. 
The unit of pathogenesis in an oyster can be a population, a consortium of populations, or a clone.

Oyster pathogenesis ultimately also involves the coevolutionary interplay between vibrios, oysters, and their microbiota.

Keywords : polymicrobial disease ; specific-pathogen-free (SPF) ; genomic ; ecology ; evolution 


\section{GLOSSARY}

- Allopatric: a set of sampled isolates or genomes from different geographic areas, between which barriers to migration and gene flow are significant.

- Clone: a group of genetically identical individuals.

- Consortium: two or microbial groups living in close association and interacting in a predictable manner.

- Core genome: the set of genes present in all members of a clade or population.

- Ecological population: a genetic unit representing a cohesive ecology, gene flow and social attributes.

- Flexible genome: the set of genes present in only a fraction of the members of a clade or population.

- Gene-specific selective sweep: the process in which an adaptive gene or allele (possibly a niche-specifying variant) spreads in a population by recombination faster than by clonal expansion. The result is that the adaptive variant is present in more than a single clonal background, and that diversity is not purged genome-wide.

- Genome-wide selective sweep: the process in which an adaptive gene or allele (possibly a niche-specifying variant) spreads in a population by clonal expansion of the genome that first acquired it. The result is that diversity is purged genome-wide, and that the adaptive variant is linked in the same clonal frame as the rest of the genome.

- Horizontal gene transfer (HGT): the incorporation of foreign DNA into a genome. Incorporation can be mediated by either homologous recombination or nonhomologous recombination of DNA that enters a cell via transformation, transduction, or conjugation.

- Microbiota: a community of microorganisms inhabiting a host.

- Polymicrobial disease: an infection of an individual by a mix (co-infection) of two or more strains, population or microbes either together or sequentially.

- Quorum sensing: a system mediated by the production of signaling molecules, inducing a genetic response correlated to population density.

- Sympatric: a set of sampled isolates or genomes from the same geographic area, within which barriers to migration and gene flow are low or nonexistent. 
- Type III survivorship curve: Species with type III survivorship curves show the highest age-specific mortality in early life stages, which is typical for most marine invertebrates including oysters. 


\section{Involvement of Vibrios in Oyster Disease}

Vibrios are ubiquitous marine bacteria that are ecologically and metabolically diverse members of planktonic and animal-associated microbial communities $[1,2]$. They have been called 'opportuni-trophs' [3] due to their high metabolic versatility and genetic variability coupled with chemotaxis and quorum sensing (see Glossary), all of which allows high colonization potential [4, 5]. Vibrios encompasses the ancient and well-studied human pathogen, $V$. cholerae, as well as some less thoroughly characterized opportunistic pathogens capable of infecting humans, including $V$. parahaemolyticus and $V$. vulnificus [2]. Even less well understood are the consequences of Vibrio infections in other animals, including fish, coral, shrimp and mollusks; however, infections in these organisms can have important environmental and economic consequences [6]. In particular, vibrios have been suggested to be responsible for repeated mortality outbreaks in oyster beds (Crassostrea gigas) in France that have resulted in losses of up to $80-100 \%$ of production (Box 1). However the onset and progression of disease in the wild has been difficult to follow in the past. Hence it is often not clear whether vibrios isolated from diseased oysters are the causative agent, secondary opportunistic colonizers, or commensals [7].

Vibrios appear to employ a diversity of molecular mechanisms to infect oysters, albeit our knowledge is mainly based on very few model strains. For example, infection with Vibrio tasmaniensis LGP32 [8] involves an intracellular phase in hemocytes, which are the oyster immune-competent cells, and resistance to (i) antimicrobial peptides (AMPs), (ii) reactive oxygen species (ROS) and (iii) copper [9-12]. Vibrio aestuarianus, on the other hand, interacts with hemocytes extracellularly and inhibits their phagocytotic abilities [13, 14]. A common theme, however, is that metalloproteases have been linked to toxicity in $V$. tasmaniensis, V. aestuarianus, Vibrio corallilyticus and Vibrio tubiashi [13, 15, 16]. Nonetheless, the disruption of this gene in $V$. tasmaniensis does not result in an attenuation of virulence [17] and presence or absence of this gene is not diagnostic for pathogenicity [18]. Finally, it should be emphasized that although these model strains were all isolated from diseased animals, they have not been found in repeated surveys. In fact, naturally infected oysters are typically colonized by a diverse assemblage of vibrios [7, 19-21] and it has been hypothesized that this diversity may contribute to pathogenesis [8]. Indeed despite the widely shared concept in veterinary medicine that the functionally active unit of pathogenesis is a single strain, numerous examples of genetically diverse pathogens or polymicrobial disease have been described [22]. 
Considering the uncertainties outlined above, it is not a priori clear what represents the functional units of pathogenesis in oyster disease. Are such units clones that have emerged after recent acquisition of virulence factors, populations of closely related organisms with virulence as a core function, or a specific consortium where virulence (or severity of pathogenesis) is an emergent property of their specific interaction? Exploring these questions is important for diagnosing, predicting and preventing disease outbreaks in aquaculture and requires understanding of the complex ecological and evolutionary dynamics of infectious agents.

In this review, we propose the oyster $C$. gigas and Vibrio pathogens as a model to explore disease dynamics in wild animals. This is now possible because of several recent developments. First, vibrios have been subject to population genetic analysis, which has allowed the delineation of functionally and genetically cohesive ecological populations. These provide a framework for mapping of disease properties combined with analysis of their environmental dynamics and interpretation of selective mechanisms. Second, specific pathogen-free (SPF) juvenile oysters have recently been developed enabling experimental infections under near natural conditions but without the potential confounding effect of prior infections by unknown environmental pathogens [23]. Finally, the development of genetic tools to manipulate non-model Vibrio strains offers a unique opportunity to demonstrate the functional role of selected genes in virulence. We show how high-resolution environmental sampling, population and functional genomics combined with high throughput infection assays can lead to identification of the functional unit of pathogenesis (Box 2). Moreover, we explore the co-evolutionary interplay between vibrios, oysters and their microbiota.

\section{Population Structure as a Framework for Analysis of Disease Dynamics}

In ecology, the term population refers to coexisting individuals of the same species, sharing a common gene pool and habitat. Importantly, a common gene pool ensures that adaptive genes can spread in a population-specific manner, making populations units of gene-flow and ecology. However, because horizontal gene flow was postulated to erode population structure among microbes [24], such textbook definition was thought to only apply to sexually reproducing eukaryotes. Indeed, the term population is used very loosely in microbiology, often referring to a range of genetic units from clonal cultures to operational taxonomic units defined by arbitrarily fixed sequence similarity cutoffs. Nonetheless, recent work has shown 
that, for microbes, it is also possible to define genetic, ecological, behavioral and social units akin to populations of plants and animals $[25,26]$. Because vibrios have served as major models in this effort, we can apply this population framework to the ecology and evolution of virulence.

Population structure in vibrios was initially explored in series of studies, which tested differential association of genotypes with potential microhabitats in the same water samples (e.g., free-living, organic particle or zooplankton associated) or animal tissues (e.g., guts, gills) (Figure 1, Key Figure) [27-29]. A major advantage of vibrios for such endeavors is that they can be easily cultured allowing multilocus or whole genome sequencing to obtain finescale genetic resolution among individual isolates. Overall, these studies revealed populations as fine-scale genotypic clusters with differential environmental distribution, often below the resolution of rRNA genes, which are typically used for microbial ecology studies [29]. For example, while some populations were predicted to be primarily associated with different types of organic particles or zooplankton, others were predominantly free-living. Subsequent work showed that these populations also represent gene flow units through which adaptive genes and alleles can sweep [30]. This indicates that they are gene flow units with recombination within exceeding that between populations [31].

An important consequence of the population framework outlined above is that it allows (i) analysis of environmental dynamics and reservoirs of potential pathogens and (ii) interpretation of the types of selection acting on pathogenicity determinant genes [32]. First, the ecology of pathogens in the water column might influence the infection dynamics. As detailed further below, oysters are filter feeders with strong preference for retention of larger particles raising the possibility that pathogens attached to particles possess higher probability of contact and hence infection [33]. Moreover, the dynamics of the population outside the oyster host may have important consequences for when and where infection is more likely to occur. This includes population blooms triggered by specific environmental conditions [1], reactivation of viable but non-cultivable (VBNC) forms [34] as well as action of predators such as grazer or phages [35-37]. Second, observed population structure may yield insight into the evolutionary history of the pathogens and types of selection acting on genes encoding pathogenicity determinants. For example if these genes are present in all members of a population, and hence represent a core function, it can be assumed that they are under strong positive selection. On the other hand, if traits are present intermittently in non-clonal strains 
within a population, they might be under frequency dependent selection [38, 39] (i.e., they may be beneficial when rare but increasingly detrimental when more common). For example, some surface structures, such as the O-antigen or pili, can act as virulence factors but they are also targets for bacterial predators such as phages and protozoa [40, 41]. These dynamics maintain variability among closely related strains by frequent exchange by horizontal gene transfer (HGT) of different variants, including frequent gene loss and addition. Finally, if pathogens are highly clonal members of an otherwise diverse population, a recent evolutionary origin might be indicated via a clonal expansion or a genome-wide selective sweep. Importantly, such evolutionary analyses will also influence monitoring strategies since populations that have pathogenicity as a core function can be detected in broader surveys by some marker gene, while more clonal pathogens most likely require targeting genes encoding pathogenicity determinants.

\section{Experimental Ecology: Closing the Gap Between Natural and Laboratory Infections}

Investigating pathogenesis requires an animal model of infection. Attempts to kill oysters by immersion in Vibrio-contaminated seawater have frequently proven unsuccessful, and have necessitated reliance on infection via injection [12]. Although mortality often appears within a few days, injection does not reflect the natural route of infection and may thus exclude important factors (e.g., chemotaxis and colonization). Moreover, as mentioned above, infection in the wild may also be enhanced by association of pathogens with zoo- or phytoplankton and organic particles (Figure 1). Vibrio populations can assemble in oysters via continuous inoculation through their filter-feeding mode of nutrition where water is being pumped at high rates through the gills. These act as a sieve capturing food particles in the optimum range of 5-10 $\mu \mathrm{m}$ and moving them toward the mouth [33]. In this process, mucus and gill associated microbiota may constitute a first defense against vibrios [42, 43]. Thus, laboratory infection of animals where cultured bacteria are simply provided in a monoclonal state is unlikely to yield an accurate understanding of the factors that contribute to virulence. Identifying the microhabitats of pathogens [29] may further facilitate development of an experimental infection model representative of the natural route (e.g., by mimicking organic particles through use of adapted polymeric substrates) and allow for a better understanding of the mode of transmission and primary tissue targets for pathogens.

The recent development of SPF juveniles of $C$. gigas constitutes an important step forward towards investigating natural infection mechanisms (Figure 2). SPF oysters are descendants of 
a pool of genitors that are produced in hatcheries under highly controlled conditions to minimize the influence of genetic and environmental parameters that could affect the host sensitivity to the disease [7, 23, 44]. SPF oysters are not axenic, as is the case with gnotobiotic animals developed to explore infectious processes without interference or influence from unknown microbiota [45]. However gnotobiotic models are restricted to laboratory studies and have not been deployed in the environment to explore the disease in the wild. Cultivable bacterial microbiota and Vibrio were shown to be about $10^{3} \mathrm{cfu} / \mathrm{mg}$ and $<1 \mathrm{cfu} / \mathrm{mg}$ of oyster tissue respectively [7]. Although the exact composition of the SPF microbiome remains unknown, it has not been observed to cause disease even in prolonged maintenance of SPF oysters. SPF oysters can be placed in the environment where the onset of mortality and cumulative mortality rates can be monitored under natural conditions (Figure 2). Once disease has been detected, live animals can be used in laboratory experiments to investigate the diversity and dynamics of microbes during disease progression. Moreover, these SPF oysters can be used for high-throughput experimental infections with hundreds of bacterial isolates $[7,21,46]$. Hence, standardized SPF oysters constitute an animal model to (i) sample naturally colonizing vibrios from the environment, (ii) allow natural progression of infection, and (iii) determine virulence mechanisms across populations or strains of infecting vibrios.

\section{Population as the Unit of Vibrio Pathogenesis}

SPF oysters have recently been used in a field based approach to investigate the disease ecology of microdiverse Vibrio genotypes in oysters [21]. Briefly, SPF oysters were exposed to natural seawater in the field during a mortality outbreak, and returned to the laboratory to determine Vibrio dynamics during disease expression (Figure 3A). This study showed that the onset of disease is associated with progressive replacement of diverse benign colonizers by members of a virulent population defined according to the criteria outlined above. This population was taxonomically assigned to the Vibrio crassostreae species [47]. Although the virulent population is genetically diverse (average nucleotide identity $\sim 98 \%$, Figure $4 \mathrm{~A}$ ), all members of that population can cause disease suggesting that virulence is a core function. Comparative genomics with a closely related population (Vibrio spp. named here $V$. crassostreae-like, Figure 4A) containing only non-virulent strains, identified genes that were only found in virulent strains and clustered in population-specific genomic regions. The importance of these loci for $V$. crassostreae virulence was investigated using a genetic knockout approach. Among 81 population-specific genes, only one (R5-7, encoding a protein 
of unknown function) was found to be necessary for $V$. crassostreae virulence. This suggests that the primary role of the majority of population-specific genes is not related to pathogenicity in oysters. Instead some of the genes were annotated as algae scavenging (e.g. arylsulfatase) and attachment (e.g. tad gene cluster) indicating perhaps a role in interaction with algal detritus outside the host.

Core genome phylogeny reveals that the virulent population ( $V$. crassostreae) and the nonvirulent population ( $V$. crassostreae-like) are very closely related to two populations (\#12 and 13) that have been isolated from seawater fractions collected at a coastal site in Massachusetts (USA) where no significant oyster farming is occurring [29] (Figure 4A). Preliminary results suggest that population \#12 is virulent while population \#13 is non-virulent (Figure 4A). Although the relevance of these populations for oyster disease has not been established in the wild, these findings match the co-occurrence of $V$. crassostreae with its non-pathogenic relatives in France. In fact, the biogeography suggests that both pathogenic populations are descendants from the same lineage, possibly having speciating by geographic isolation. In addition, the populations from the US coastal site provide another example of coincidence of ecological population delineation with virulence. The close relationship between virulent and non-virulent populations demonstrates the importance of fine-scale differentiation, below the resolution level obtained by traditional marker gene approaches, in assessing disease risk in the environment.

Several other studies assessing the distribution of vibrios in animals also support environmental dynamics as an important factor in colonization. Surprisingly low specificity for animals was suggested by comparison of Vibrio populations collected from seawater and different body regions of mussels and crabs [28]. Importantly, for mussels, which can retain very small bacteria-sized particles in their filtering apparatus, populations from the water column colonized the animals at similar frequencies suggesting very little selectivity. Similarly, neutrality of the association between Vibrio and oysters was also suggested in $C$. gigas [19] although in this study phylogenetic and ecological resolution may not have been sufficient to delineate populations. In addition, no comparison of the Vibrio distribution between oyster and seawater was performed and the sampling site had no recorded oyster mass mortalities [48]. Hence in the future, natural infections of SPF oysters in areas affected by mass mortalities may constitute a suitable system to investigate connectivity between hosts and environmental reservoirs. This may allow to specifically test to what extent oysters are a 
selective habitat for Vibrio populations and which specific Vibrio genotypes are favored during pathogenesis. In this endeavor, a key first step is the identification of the unit of pathogenesis, which is a genotypically diverse population in the case of $V$. crassostreae but, as detailed below, can also be a clone or consortium.

\section{A Consortium as the Unit of Pathogenesis}

Several lines of evidence suggest that the oyster juvenile disease is enhanced by microbial interactions. First, experimental infections have demonstrated that some strains are moderately virulent when injected into animals individually, and display heightened virulence in mixed experimental infections [49]. Second, investigation of population dynamics during oyster infection have shown that naturally infected oysters initially contain a large proportion of diverse non-virulent strains, but that these are progressively replaced by a virulent population rising to $\sim 50 \%$ of the bacterial isolates at the point of maximal cumulative mortalities (Figure 3A) [21]. To address the role of non-virulent strains in the infection process, a virulent strain (V. crassostreae J2-9) was injected into oysters at various doses, either alone or in combination with non-virulent strains (Vibrio sp. or Shewanella sp.) (Figure 3B). When injected alone, reduction of the injected dose of the virulent strain by dilution in the culture media significantly reduced oyster mortality. In contrast, when the virulent strain was injected at low dose with high doses of non-virulent strains, mortality rates were markedly increased. Thus, the presence of non-virulent bacteria may dramatically increases the virulence of $V$. crassostreae at the onset of infection, suggesting that there are effects of bacterial density upon virulence, irrespective of the genotype of the co-infecting strain. Hence, although nonvirulent strains are not sufficient for pathogenesis, they clearly contribute to virulence. One possibility is that non-virulent strains generate sufficiently high bacterial loads to either overcome host defenses or to induce expression of virulence factors via quorum sensing [50].

Further exploration of the dynamics of infection in individual oysters may show whether sequential or simultaneous infections by diverse Vibrio population assemble randomly or by cooperative interactions. A non-random association could mean that each population has a distinct functional role in the pathogenesis, such as the production of a siderophore, exoprotease, antimicrobial molecules or quorum sensing autoinducer.

\section{Clones as the Functional Unit of Pathogenesis}

A dramatic increase of oyster mortalities at the adult stage has recently been associated with 
the presence of $V$. aestuarianus (Box 1). Although preliminary, recent phylogenomic analysis has suggested that the functional unit of $V$. aestuarianus pathogenesis are clones [46]. Strains isolated from diseased oysters cluster into two nearly identical clonal lineages whereas strains isolated from healthy oysters, cockles or zooplankton were found to be more diverse (Figure $4 \mathrm{~B}, \mathrm{C})$. The majority of strains within each of the two clonal lineages are virulent, suggesting that a few strains have recently lost their pathogenicity. Consistent with this notion, a single nucleotide deletion was linked to non-virulence in one strain. This mutation induces a frame shift in the $\operatorname{var} S$ gene that codes for the transduction histidine-protein kinase of the sensory system VarS/VarA [51]. The varS gene was intact in the other strains and genetic analyses confirmed that it is necessary for infection of oysters and for expression of a secreted metalloprotease [46].

The pathogenic clonal lineages of $V$. aestuarianus appear phylogenetically distinct from their closest conspecifics, suggesting that a recent clonal sweep or speciation event has differentiated them from their parental population (Figure 4B, C) [46]. However, the population structure remains poorly resolved, since sampling has to date been highly biased towards moribund animals. In fact, knowledge of the ecology of these bacteria is sparse and stems largely from laboratory experiments using single strains in experimental microcosms [52] or surveys covering large spatio-temporal scales with limited taxonomic resolution [53]. If indeed these pathogens adhere to the more classical view that clones are the units of pathogenesis it will be very interesting to compare their ecological and evolutionary dynamics to $V$. crassostreae, which displays genotypically diverse infections (Box 2).

\section{Incorporating Higher Order Interactions}

No matter if the unit of infection is a clone (e.g., V. aestuarianus [46]), a population (e.g., V. crassostreae [21] or a consortium [49], biotic interactions with other host-associated microbes adds another level of complexity. For example specific states of host microbiomes may be more resistant to invasion by pathogens. Yet microbiomes may be altered by infecting populations and may also respond to evolutionary changes at the level of host genotypes as a result of selection exerted by pathogen infections. Hence an eco-evolutionary process may ensue that needs to be explored in order to fully understand disease dynamics in the wild.

\section{Interaction With the Microbiome}


In many microbial systems, the relevant unit for many functions is neither a clone nor population but consortia living in close symbiotic association $[54,55]$. These microbiota fulfill a range of different functions with defense against pathogens being among the most prominent in humans [56, 57], corals [58] but also oysters [59]. Microbial assemblages can vary substantially between oyster tissues [60]. Microbiota in gills have been found to be stable across steep environmental gradients [61] and gill microbial community composition also correlated to oyster genotype [62] suggesting co-evolutionary relationships between host and microbiota but not necessarily with Vibrio populations. These genotype specific assemblies of microbiota were however vulnerable to environmental disturbance [62] indicating that abiotic stress can destabilize microbiome assemblies. Abiotic environmental factors also strongly influence the dynamics of hemolymph microbiota by determining turnover rates as well as inter-individual differences. Importantly, oysters that are associated with more stable communities are more resistant to invasion by pathogens [63].

Oyster hemolymph also shows the highest Vibrio load in comparison to other tissues, but mortalities under field conditions were only observed when the Vibrio load was also high in other tissues [42] suggesting that the spillover of virulent Vibrio populations from the hemolymph to solid tissues causes systemic disease. Interestingly, in hemolymphs of experimentally infected moribund animals, bacterial communities disturbed by Vibrio infection were not dominated by the Vibrio strain used for infection, but by unrelated secondary opportunists (e.g., Arcobacter) that profited from the initial community disturbance induced by the infection [63]. This stresses the right timing and tissue choice for sampling since moribund animals may be dominated by such secondary opportunists masking the causative shifts in Vibrio and other microbial population during the onset of disease. It further highlights the importance of the hemolymph as the key body compartment for the interaction among Vibrio populations, the host and other components of the microbiome. The degree in which different tissues are connected to the environment in conjunction with tissue specificity of microbiota [42] suggest different colonization barriers that should be considered in the complex turnover dynamics of Vibrio populations against the background of disease.

\section{Evolutionary Responses of the Host}

Any attempt to understand the emergence of disease needs to consider the prior adaptations and environment dependent physiological responses of the host. Whenever disease is a strong regulator of host populations, selection for host resistance will also be strong [64, 65]. In 
species with type III survivorship curves with high reproductive output and high mortalities in early life stages like the Pacific oyster, selection will be particularly efficient at the dispersing larval stage $[65,66]$ whereas the adaptive potential in adults might be more limited.

In larvae, cross-infection experiments exposing oyster hosts from genetically differentiated natural oyster stocks [67] to sympatric and allopatric vibrios showed lower mortality in sympatric combinations [68]. Resistance against infection by local strains demonstrates that hosts have the adaptive potential to rapidly respond to selection exerted by disease. This effect was observed across 76 sympatric and allopatric Vibrio strains representing several clades of the Vibrio phylogeny. The widespread occurrence of local host resistance against otherwise distantly related strains indicates the presence of common targets for host adaptation in all strains. Rather than by common descent, the genetic basis of these targeted traits in the Vibrio strains can be explained by either local sharing of genetic elements within Vibrio populations or parallel evolution as a response to similar selection pressures [68]. The selective pressures leading to these geographic rather than phylogenetic matches remain unknown, but it seems likely that they result from adaptations to living associated with oyster hosts, making the genes responding to selection a suitable target for the evolution of host resistance.

On the host side, resistance against sympatric strains displayed dominant inheritance in crosses between both oyster stocks, suggesting a comparatively simple genetic architecture of resistance. Due to the genomic resources available for oysters (Box 2), the identification of the underlying molecular mechanisms becomes a tractable goal that will help to understand the selective landscapes shaping the interactions between oyster hosts and their colonizing Vibrio populations. A similar response of natural host resistance was not yet observed at sites with recurrent Vibrio-associated disease in juveniles. This might suggest a lower evolutionary potential due to lower genetic diversity of farmed animals [69] in combination with divergent selection pressures exerted by diverse sets of virulence genes in virulent Vibrio populations. Additionally, high densities and repeated transport of farmed oysters can result in larger reservoirs of virulent vibrios with more efficient transmission dynamics leading to rapid, synchronized shifts towards virulent Vibrio populations. Such population shifts may further prevent an efficient evolutionary response of the hosts. Production of SPF oysters from disease-free stocks and diseased stocks can be used to link natural exposure in field 
experiments to experimental infections using the corresponding contrasting Vibrio populations [7]. The evolutionary dynamics of hosts and Vibrio populations observed in such studies can then serve to investigate the different modes of disease emergence on all levels of interactions between hosts and the biotic environment (vibrios and microbiota).

\section{Concluding Remarks}

As we have outlined in this review, the interaction between oysters and vibrios is an emerging model system to address new and original scientific questions concerning the dynamics of infectious diseases in the wild and its feedback on ecology and evolution of host-pathogen interactions. Ecological populations often represent the functional unit of pathogenesis, which does not preclude that there can be cases where the causal agent is a single clone. In either case disease cannot be understood in isolation from the host and its biotic and abiotic environment. Holistic approaches taking the complexity of all levels of these interactions into account are needed in the future (see Outstanding Questions) to deepen our understanding of disease processes and provide tools for an efficient management of disease in the wild.

\section{Acknowledgements}

We warmly thank Professor Brian Austin (University of Stirling, UK) for his help in editing the language of our manuscript. We thank Dr Maxime Bruto for providing unpublished phylogenetic trees and Bruno Petton and Dr Stéphane Pouvreau for nicely providing the pictures. The present study has been supported by the ANR (11-BSV7-023-01 «VIBRIOGEN », 13-ADAP-0007-01« OPOPOP»). 


\section{References}

1. Takemura, A.F., D.M. Chien, and M.F. Polz, Associations and dynamics of Vibrionaceae in the environment, from the genus to the population level. Frontiers in microbiology, 2014. 5: p. 38.

2. Thompson, F.L., T. Iida, and J. Swings, Biodiversity of vibrios. Microbiol Mol Biol Rev, 2004. 68(3): p. 403-31, table of contents.

3. Polz, M.F., et al., Patterns and mechanisms of genetic and phenotypic differentiation in marine microbes. Philosophical transactions of the Royal Society of London. Series B, Biological sciences, 2006. 361(1475): p. 2009-21.

4. Reen, F.J., et al., The genomic code: inferring Vibrionaceae niche specialization. Nat Rev Microbiol, 2006. 4(9): p. 697-704.

5. Hazen, T.H., et al., The contribution of mobile genetic elements to the evolution and ecology of Vibrios. FEMS Microbiol Ecol, 2010. 74(3): p. 485-99.

6. Le Roux, F., et al., The emergence of Vibrio pathogens in Europe: ecology, evolution, and pathogenesis (Paris, 11-12th March 2015). Front Microbiol, 2015. 6: p. 830.

7. Petton, B., et al., Crassostrea gigas mortality in France: the usual suspect, a herpes virus, may not be the killer in this polymicrobial opportunistic disease. Front Microbiol, 2015. 6: p. 686.

8. Le Roux, F., et al., Genome sequence of Vibrio splendidus: an abundant planctonic marine species with a large genotypic diversity. Environ Microbiol, 2009. 11(8): p. 1959-70.

9. Vanhove, A.S., et al., Outer membrane vesicles are vehicles for the delivery of Vibrio tasmaniensis virulence factors to oyster immune cells. Environ Microbiol, 2014. 17(4): p. 1152-65.

10. Vanhove, A.S., et al., Copper homeostasis at the host vibrio interface: lessons from intracellular vibrio transcriptomics. Environ Microbiol, 2015. doi: 10.1111/14622920.13083

11. Duperthuy, M., et al., The major outer membrane protein OmpU of Vibrio splendidus contributes to host antimicrobial peptide resistance and is required for virulence in the oyster Crassostrea gigas. Environ Microbiol. 12(4): p. 951-63.

12. Duperthuy, M., et al., Use of OmpU porins for attachment and invasion of Crassostrea gigas immune cells by the oyster pathogen Vibrio splendidus. Proceedings of the National Academy of Sciences of the United States of America, 2011. 108(7): p. 2993-8. 
13. Labreuche, Y., et al., Vibrio aestuarianus zinc metalloprotease causes lethality in the Pacific oyster Crassostrea gigas and impairs the host cellular immune defenses. Fish \& shellfish immunology, 2010. 29(5): p. 753-8.

14. Labreuche, Y., et al., Effects of extracellular products from the pathogenic Vibrio aestuarianus strain 01/32 on lethality and cellular immune responses of the oyster Crassostrea gigas. Dev Comp Immunol, 2006. 30(4): p. 367-79.

15. Binesse, J., et al., Metalloprotease vsm is the major determinant of toxicity for extracellular products of Vibrio splendidus. Appl Environ Microbiol, 2008. 74(23): p. 7108-17.

16. Hasegawa, H., et al., The extracellular metalloprotease of Vibrio tubiashii is a major virulence factor for pacific oyster (Crassostrea gigas) larvae. Appl Environ Microbiol, 2008. 74(13): p. 4101-10.

17. Le Roux, F., et al., Construction of a Vibrio splendidus mutant lacking the metalloprotease gene vsm by use of a novel counterselectable suicide vector. Appl Environ Microbiol, 2007. 73(3): p. 777-84.

18. Saulnier, D., et al., A large-scale epidemiological study to identify bacteria pathogenic to Pacific oyster Crassostrea gigas and correlation between virulence and metalloprotease-like activity. Microb Ecol, 2010. 59(4): p. 787-98.

19. Wendling, C.C., F.M. Batista, and K.M. Wegner, Persistence, seasonal dynamics and pathogenic potential of Vibrio communities from pacific oyster hemolymph. PLoS One, 2014. 9(4): p. e94256.

20. Gay, M., F.C. Berthe, and F. Le Roux, Screening of Vibrio isolates to develop an experimental infection model in the Pacific oyster Crassostrea gigas. Diseases of aquatic organisms, 2004. 59(1): p. 49-56.

21. Lemire, A., et al., Populations, not clones, are the unit of vibrio pathogenesis in naturally infected oysters. ISME J, 2014. 9(7): p. 1523-31.

22. Methot, P.O. and S. Alizon, What is a pathogen? Toward a process view of hostparasite interactions. Virulence, 2014. 5(8): p. 775-85.

23. Petton, B., et al., Temperature influence on pathogen transmission and subsequent mortalities in juvenile Pacific oysters Crassostrea gigas. Aquacult Environ Interact 2013. 3: p. 257-273.

24. Papke, R.T., et al., Searching for species in haloarchaea. Proc Natl Acad Sci U S A, 2007. 104(35): p. 14092-7. 
25. Shapiro, B.J. and M.F. Polz, Ordering microbial diversity into ecologically and genetically cohesive units. Trends Microbiol, 2014. 22(5): p. 235-47.

26. Shapiro, B.J. and M.F. Polz, Microbial Speciation. Cold Spring Harb Perspect Biol, 2015. 7(10): p. a018143.

27. Szabo, G., et al., Reproducibility of Vibrionaceae population structure in coastal bacterioplankton. ISME J, 2012. 7(3): p. 509-19.

28. Preheim, S.P., et al., Metapopulation structure of Vibrionaceae among coastal marine invertebrates. Environ Microbiol, 2011. 13(1): p. 265-75.

29. Hunt, D.E., et al., Resource partitioning and sympatric differentiation among closely related bacterioplankton. Science, 2008. 320(5879): p. 1081-5.

30. Shapiro, B.J., et al., Population genomics of early events in the ecological differentiation of bacteria. Science, 2012. 336(6077): p. 48-51.

31. Polz, M.F., E.J. Alm, and W.P. Hanage, Horizontal gene transfer and the evolution of bacterial and archaeal population structure. Trends Genet, 2013. 29(3): p. 170-5.

32. Cordero, O.X. and M.F. Polz, Explaining microbial genomic diversity in light of evolutionary ecology. Nat Rev Microbiol, 2014. 12(4): p. 263-73.

33. Froelich, B., M. Ayrapetyan, and J.D. Oliver, Vibrio vulnificus integration into marine aggregates and subsequent uptake by the oyster, Crassostrea virginica. Appl Environ Microbiol, 2012. 78(11): p. 3885-9.

34. Froelich, B.A. and R.T. Noble, Factors affecting the uptake and retention of Vibrio vulnificus in oysters. Appl Environ Microbiol, 2014. 80(24): p. 7454-9.

35. Chen, H. and H.N. Williams, Sharing of prey: coinfection of a bacterium by a virus and a prokaryotic predator. MBio, 2012. 3(2): p. e00051-12.

36. Hilbi, H., et al., Environmental predators as models for bacterial pathogenesis. Environ Microbiol, 2007. 9(3): p. 563-75.

37. Lutz, C., et al., Environmental reservoirs and mechanisms of persistence of Vibrio cholerae. Front Microbiol, 2013. 4: p. 375.

38. Cordero, O.X., et al., Public good dynamics drive evolution of iron acquisition strategies in natural bacterioplankton populations. Proceedings of the National Academy of Sciences of the United States of America, 2012. 109(49): p. 20059-64.

39. Cordero, O.X., et al., Ecological populations of bacteria act as socially cohesive units of antibiotic production and resistance. Science, 2012. 337(6099): p. 1228-31.

40. Seed, K.D., et al., Evolutionary consequences of intra-patient phage predation on microbial populations. Elife, 2014. 3: p. e03497. 
41. Wildschutte, H., et al., O-antigen diversity and lateral transfer of the wbe region among Vibrio splendidus isolates. Environ Microbiol, 2010. 12(11): p. 2977-87.

42. Lokmer, A., et al., The Role of Tissue-specific Microbiota in Initial Establishment Success of Pacific oysters. Environ Microbiol, 2015. doi: 10.1111/1462-2920.13163.

43. Pales Espinosa, E., A. Koller, and B. Allam, Proteomic characterization of mucosal secretions in the eastern oyster, Crassostrea virginica. J Proteomics, 2016. 132: p. 6376.

44. Petton, B., et al., Factors influencing disease-induced mortality of Pacific oysters Crassostreae gigas. Aquaculture Environ interact, 2015. 6: p. 205-222.

45. Marques, A., et al., Gnotobiotically grown aquatic animals: opportunities to investigate host-microbe interactions. J Appl Microbiol, 2006. 100(5): p. 903-18.

46. Goudenege, D., et al., A single regulatory gene is sufficient to alter Vibrio aestuarianus pathogenicity in oysters. Environ Microbiol, 2015. 17(11): p. 4189-99.

47. Faury, N., et al., Vibrio crassostreae sp. nov., isolated from the haemolymph of oysters (Crassostrea gigas). Int J Syst Evol Microbiol, 2004. 54(Pt 6): p. 2137-40.

48. Watermann, B.T., et al., Pathology and mass mortality of Pacific oysters, Crassostrea gigas (Thunberg), in 2005 at the East Frisian coast, Germany. J Fish Dis, 2008. 31(8): p. 621-30.

49. Gay, M., et al., Two vibrio splendidus related strains collaborate to kill Crassostrea gigas: taxonomy and host alterations. Diseases of aquatic organisms, 2004. 62(1-2): p. $65-74$.

50. Bassler, B.L., Small talk. Cell-to-cell communication in bacteria. Cell, 2002. 109(4): p. 421-4.

51. Lenz, D.H., et al., CsrA and three redundant small RNAs regulate quorum sensing in Vibrio cholerae. Mol Microbiol, 2005. 58(4): p. 1186-202.

52. Azandegbe, A., et al., Occurrence and seasonality of Vibrio aestuarianus in sediment and Crassostrea gigas haemolymph at two oyster farms in France. Dis Aquat Organ, 2010. 91(3): p. 213-21.

53. Vezzulli, L., et al., Aquatic ecology of the oyster pathogens Vibrio splendidus and Vibrio aestuarianus. Environ Microbiol, 2015. 17(4): p. 1065-80.

54. Giaouris, E., et al., Intra- and inter-species interactions within biofilms of important foodborne bacterial pathogens. Front Microbiol, 2015. 6: p. 841.

55. Kolenbrander, P.E., et al., Communication among oral bacteria. Microbiol Mol Biol Rev, 2002. 66(3): p. 486-505, table of contents. 
56. Abt, M.C. and E.G. Pamer, Commensal bacteria mediated defenses against pathogens. Curr Opin Immunol, 2014. 29: p. 16-22.

57. Kamada, N., et al., Control of pathogens and pathobionts by the gut microbiota. Nat Immunol, 2013. 14(7): p. 685-90.

58. Rypien, K.L., J.R. Ward, and F. Azam, Antagonistic interactions among coralassociated bacteria. Environ Microbiol, 2010. 12(1): p. 28-39.

59. Desriac, F., et al., Exploring the hologenome concept in marine bivalvia: haemolymph microbiota as a pertinent source of probiotics for aquaculture. FEMS Microbiol Lett, 2014. 350(1): p. 107-16.

60. King, G.M., et al., Analysis of stomach and gut microbiomes of the eastern oyster (Crassostrea virginica) from coastal Louisiana, USA. PLoS One, 2012. 7(12): p. e51475.

61. Zurel, D., et al., Composition and dynamics of the gill microbiota of an invasive IndoPacific oyster in the eastern Mediterranean Sea. Environ Microbiol, 2011. 13(6): p. 1467-76.

62. Wegner, K.M., et al., Disturbance induced decoupling between host genetics and composition of the associated microbiome. BMC Microbiol, 2013. 13: p. 252.

63. Lokmer, A. and K. Mathias Wegner, Hemolymph microbiome of Pacific oysters in response to temperature, temperature stress and infection. ISME J, 2015. 9(3): p. 67082.

64. Partridge, L. and P.H. Harvey, The ecological context of life history evolution. Science, 1988. 241(4872): p. 1449-55.

65. Hedgecock, Is gene flow from pelagic larval dispersal important in the adaptation and evolution of marine-invertebrate. . Bull Mar Sci, 1986. 39: p. 550-564.

66. Narvaez, D.A., J. M. Klinck, E. E. Hofmann, E. Powell, J. Wilkin, B. Haidvogel and D. Hedgecock, Effects of larval dispersion on the movement of disease resistant genes between oyster populations. Journal of Shellfish Research 2011. 30(2): p. 537-538.

67. Moehler, J., K. M. Wegner, K. Reise and S. Jacobsen, Invasion genetics of Pacific oyster Crassostrea gigas shaped by aquaculture stocking practices. Journal of Sea Research 2011. 66(3): p. 256-262.

68. Wendling, C.C. and K.M. Wegner, Adaptation to enemy shifts: rapid resistance evolution to local Vibrio spp. in invasive Pacific oysters. Proc Biol Sci, 2015. 282(1804): p. 20142244. 
69. Hedgecock, D.a.F.S., Genetic drift and effective populations sizes of hatcherypropagated stocks of the pacific oyster, Crassostrea gigas. Aquaculture, 1990. 88(1): p. 21-38.

70. Chaney, M.L. and A.Y. Gracey, Mass mortality in Pacific oysters is associated with a specific gene expression signature. Mol Ecol, 2011. 20(14): p. 2942-54.

71. Sugumar, G., et al., Vibrio splendidus biovar II as the causative agent of bacillary necrosis of Japanese oyster Crassostrea gigas larvae. Dis Aquat Organ, 1998. 33(2): p. 111-8.

72. Elston, R.A., et al., Re-emergence of Vibrio tubiashii in bivalve shellfish aquaculture: severity, environmental drivers, geographic extent and management. Dis Aquat Organ, 2008. 82(2): p. 119-34.

73. Richards, G.P., et al., Mortalities of Eastern and Pacific oyster Larvae caused by the pathogens Vibrio coralliilyticus and Vibrio tubiashii. Appl Environ Microbiol, 2015. 81(1): p. 292-7.

74. Samain, Summer mortality of Pacific oyster Crassostrea gigas. The Morest Project, Editions Quae, 379p, 2008.

75. Renault, T., et al., Concomitant herpes-like virus infections in hatchery-reared larvae and nursery-cultured spat Crassostrea gigas and Ostrea edulis. Dis Aquat Organ, 2000. 42(3): p. 173-83.

76. Lacoste, A., et al., A Vibrio splendidus strain is associated with summer mortality of juvenile oysters Crassostrea gigas in the Bay of Morlaix (North Brittany, France). Dis Aquat Organ, 2001. 46(2): p. 139-45.

77. Martenot, C., et al., Detection of different variants of Ostreid Herpesvirus 1 in the Pacific oyster, Crassostrea gigas between 2008 and 2010. Virus Res, 2011. 160(1-2): p. 25-31.

78. Renault, T., A review of mortality outbreaks in the Pacific oyster, Crassostrea gigas, reported since 2008 in various European Union Member States and the related implementation of Council Directive 2008/88/EC. Bulletin OIE, 2011. 4: p. 51-52.

79. Travers, M.A., et al., Bacterial diseases in marine bivalves. J Invertebr Pathol, 2015. 131: p. 11-31.

80. Rohfritsch, A., et al., Population genomics shed light on the demographic and adaptive histories of European invasion in the Pacific oyster, Crassostrea gigas. Evol Appl, 2013. 6(7): p. 1064-78. 
81. Sawabe, T., et al., Updating the Vibrio clades defined by multilocus sequence phylogeny: proposal of eight new clades, and the description of Vibrio tritonius sp. nov. Frontiers in microbiology, 2013. 4: p. 414.

82. Le Roux, F., B.M. Davis, and M.K. Waldor, Conserved small RNAs govern replication and incompatibility of a diverse new plasmid family from marine bacteria. Nucleic Acids Res, 2011. 39(3): p. 1004-13.

83. Hedgecock, D., et al., Second-Generation Linkage Maps for the Pacific Oyster Crassostrea gigas Reveal Errors in Assembly of Genome Scaffolds. G3 (Bethesda), 2015. 5(10): p. 2007-19.

84. Zhang, G., et al., The oyster genome reveals stress adaptation and complexity of shell formation. Nature, 2012. 490(7418): p. 49-54.

85. Sussarellu, R., et al., Additive transcriptomic variation associated with reproductive traits suggest local adaptation in a recently settled population of the Pacific oyster, Crassostrea gigas. BMC Genomics, 2015. 16(1): p. 808.

86. Fabioux, C., et al., In vivo RNA interference in oyster--vasa silencing inhibits germ cell development. FEBS J, 2009. 276(9): p. 2566-73.

87. Degremont, L., C. Garcia, and S.K. Allen, Jr., Genetic improvement for disease resistance in oysters: A review. J Invertebr Pathol, 2015. 131: p. 226-41.

88. Bachere, E., et al., Insights into the anti-microbial defense of marine invertebrates: the penaeid shrimps and the oyster Crassostrea gigas. Immunological reviews, 2004. 198: p. $149-68$.

89. Schmitt, P., et al., Molecular diversity of antimicrobial effectors in the oyster Crassostrea gigas. BMC Evol Biol, 2010. 10: p. 23.

90. Schmitt, P., et al., Insight into invertebrate defensin mechanism of action: oyster defensins inhibit peptidoglycan biosynthesis by binding to lipid II. J Biol Chem, 2010. 285(38): p. 29208-16. 


\section{Figure 1. A Model for Oyster Colonization by Vibrios.}

In seawater, vibrios co-exist as closely related, ecologically differentially associated populations (white: free living; blue and red: particle-associated; black: animal-associated), [29]. These populations may harbor virulence genes (vibrio in red) hence serving as reservoirs of pathogens [21]. To successfully colonize the host, cells must be taken up through the gills and be able to evade the immune system (large grey cell: hemocyte; orange crosses: immune effectors such as antimicrobial peptides) as well as compete with resident bacteria (colored microcells: microbiota)[19, 63]. Evidence also suggests that cooperation between virulent and non-virulent populations may enhance pathogenicity, especially in the early stages of colonization [21, 49]. Mutualistic and antagonistic interactions are show by solid and dotted thin arrows, respectively.

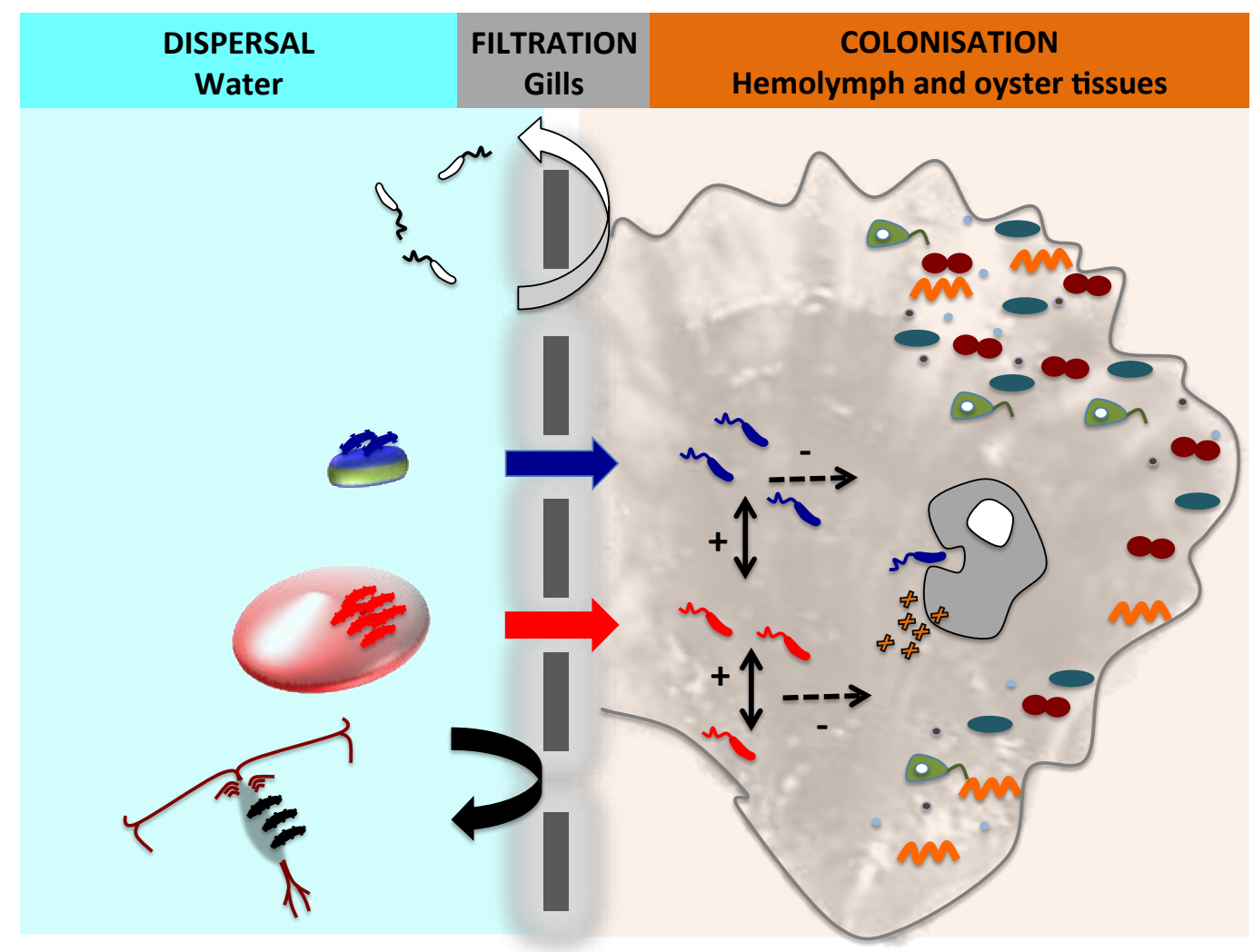




\section{Figure 2. Experimental Ecological Tools and Design.}

Specific pathogen-free (SPF) oysters are hatchery-produced descendants of a defined pool of genitors that are raised under pathogen-free conditions (for details see [23]). (A) The Crassostrea gigas life cycle comprises a pelagic and a benthic phase where larval metamorphosis is followed by attachment to a substrate. The juveniles are raised under controlled conditions, i.e., UV treated seawater, stable temperature and high nutritional value algal feeding regime. (B) SPF juvenile oysters [low Vibrio load, absence of detectable ostreid herpes virus (OsHV-1)] can be used in the natural environment as sentinels to monitor the mortality rates during a disease outbreak. Infected animals (donors) can also be brought back to the laboratory where they are incubated with SPF oysters (recipients) to investigate detailed the dynamics of infection. This links the natural infection process experienced in the field to controllable yet realistic laboratory experiments. (Pictures courtesy of Stéphane Pouvreau and Bruno Petton).

A

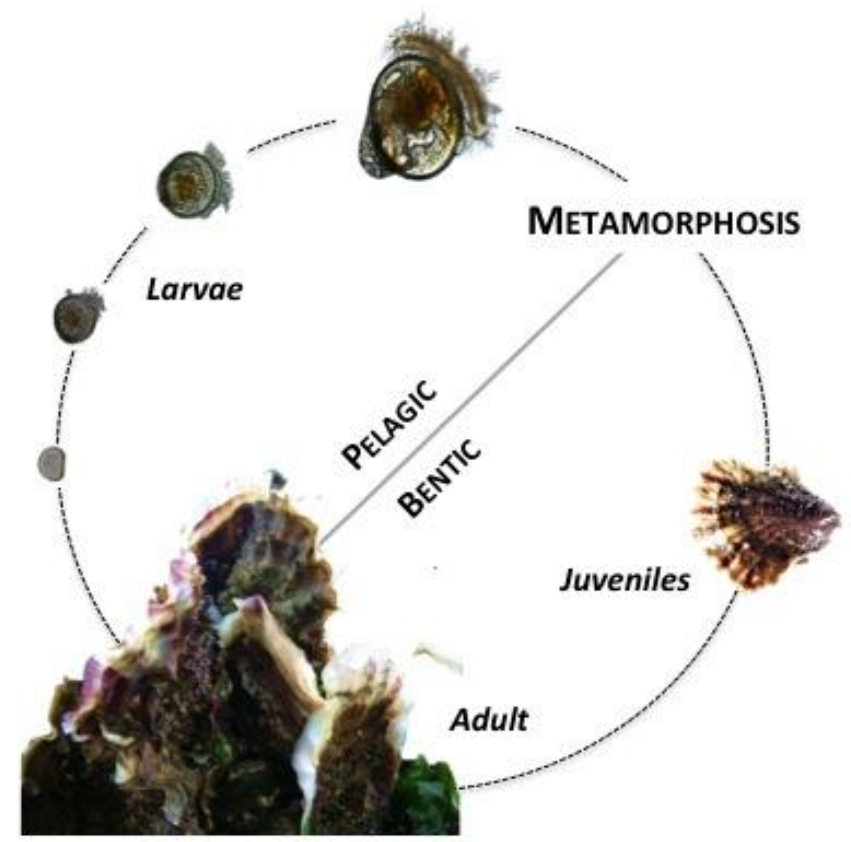

B

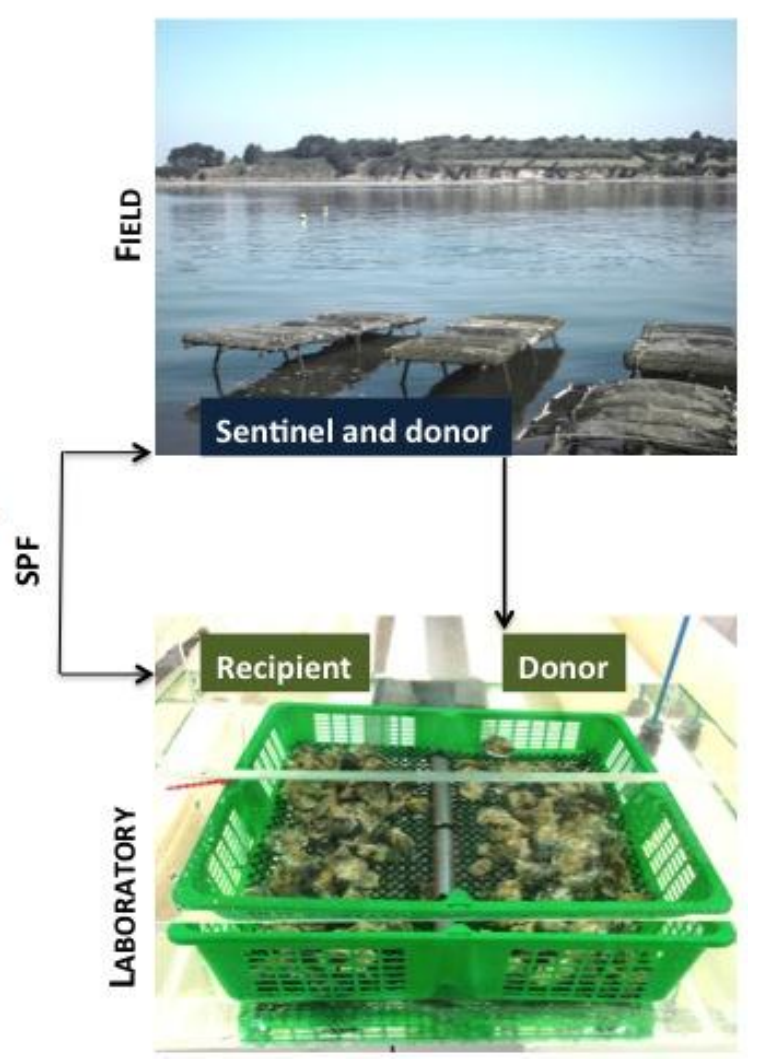


Figure 3. Co-existence of Vibrio Populations May Contribute to Virulence in Infected Oysters. (A) SPF oysters naturally infected in the field and then transferred to the laboratory to study Vibrio dynamics during disease expression. Mortalities (grey bar, y-axis) were recorded daily (day 0 to 5 , $\mathrm{x}$-axis) and expressed as percentages. Lines indicate the percentage of strains isolated from oysters belonging to non-virulent (plain and dashed blue) or virulent (red) populations (y-axis). (B) The virulent strain (J2-9, V. crassostreae, in red) was intramuscularly injected into oysters in pure culture, diluted in culture media (orange) or diluted with a pure culture of non-virulent strains (blue). The negative control is a pure culture of non-virulent strain, which was also injected. The proportion of virulent, non-virulent strains and media in the injected mix is illustrated by the colored circles. Mortality (grey bar, y-axis) was assessed after 24 hours. These graphs are adapted from [21].

A

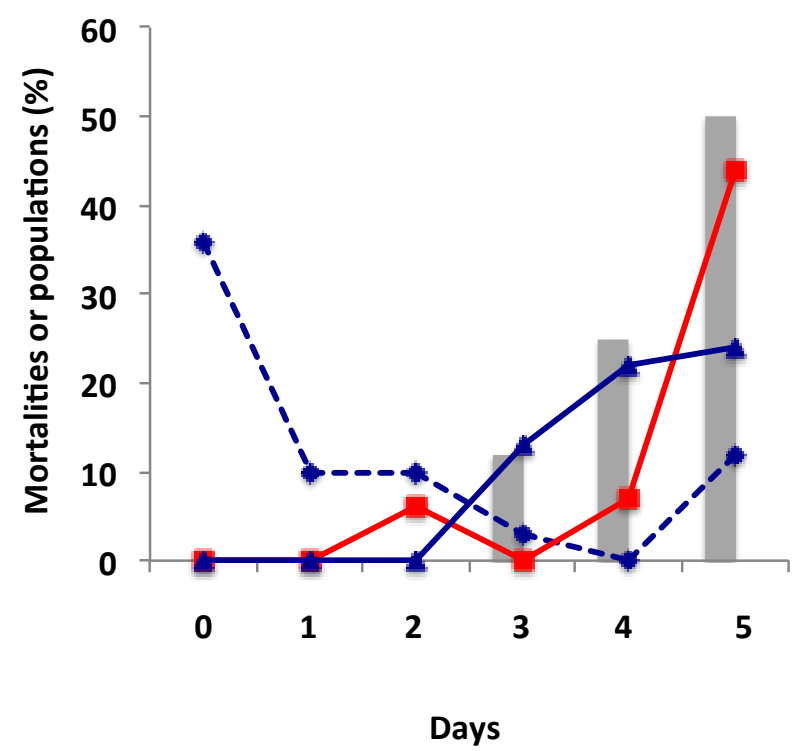

B

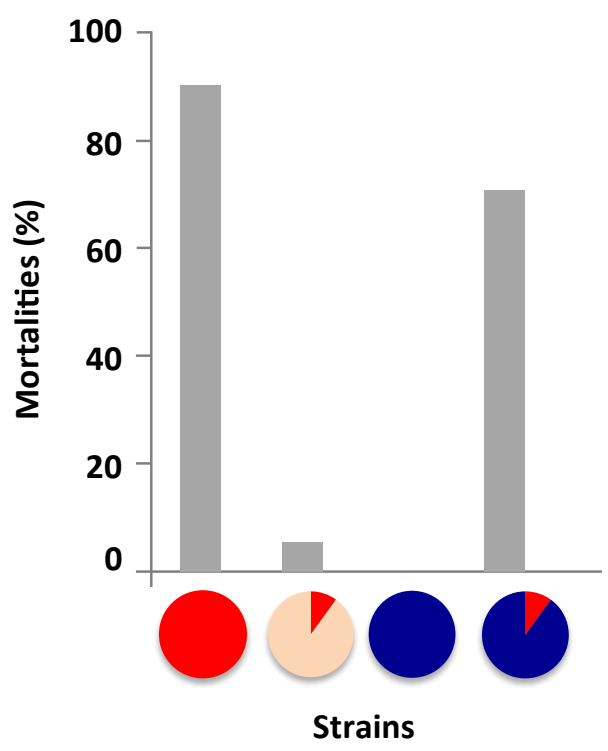


Figure 4. Phylogenetic Analysis Based on the Core Genome Reveals the Genetic Diversity of Virulent Strains in V. crassostreae (A) and the Clonality of $V$. aestuarianus (B).

The phylogenetic analysis was based on concatenated alignments of core protein sequences for $V$. crassostreae with closely related populations (population 12 and 13, Vibrio sp.) (A) and V. aestuarianus (B) (Maximum Likehood, courtesy of Maxime Bruto). V. tasmaniensis (A) and Vibrio ordalii (B) strains were used as an outgroup. A nucleic acid-based phylogenetic analysis of core genes in V. aestuarianus is shown in (C) (from [46]. Strains indicated by red and blue circles have been demonstrated by oyster injection to be virulent and non-virulent respectively. The screening of a larger collection of $V$. aestuarianus demonstrated virulence for $93 \%$ and $79 \%$ of the strains belonging to clade a and $b$, respectively while all $V$. aestuarianus isolates from healthy oysters or other invertebrates were non-virulent and phylogenetically more diverse $(C)$.

A

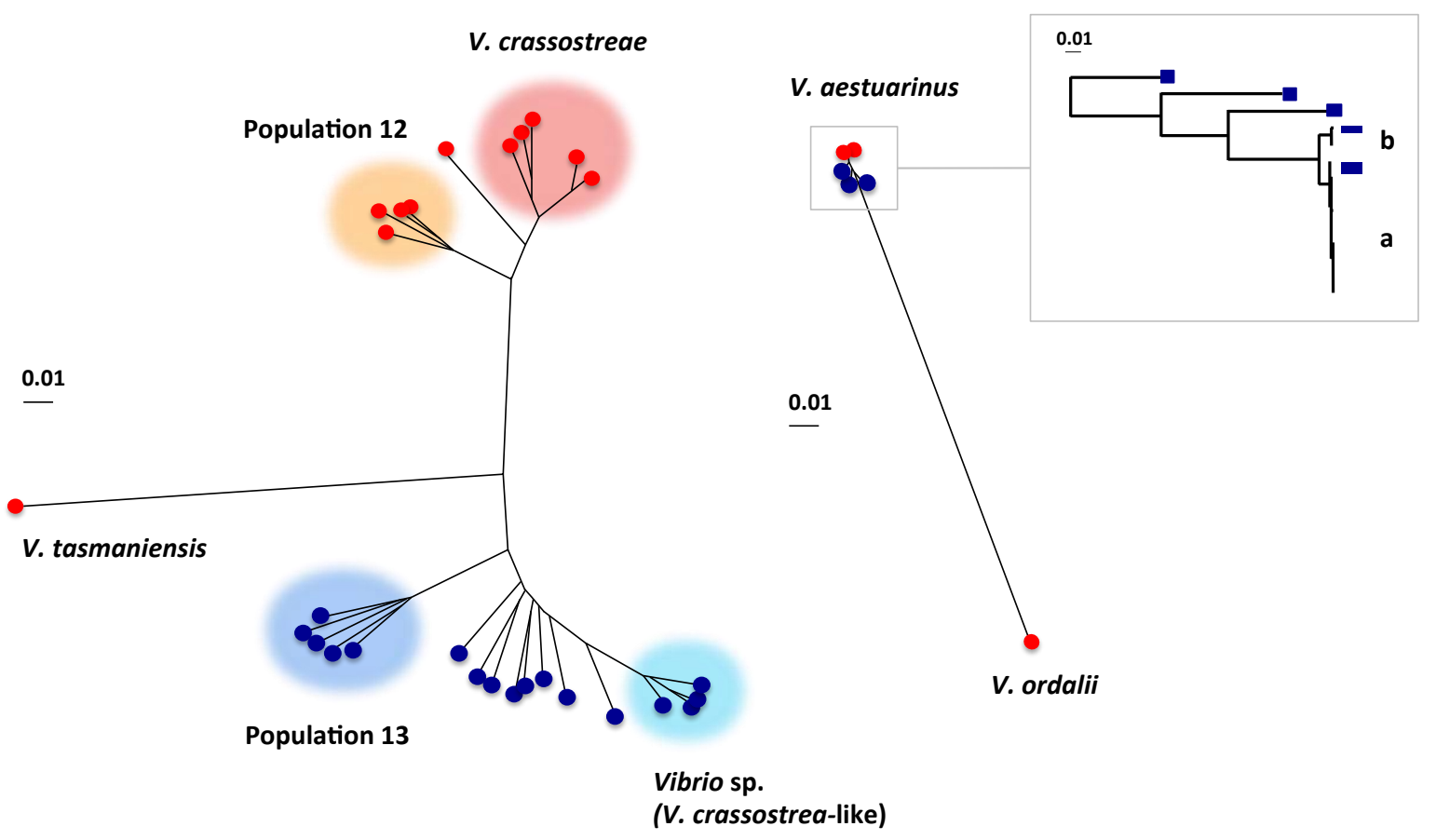




\section{Box 1. Mortality Outbreaks in Crassostrea gigas}

Due to its potential for rapid growth and a wide range of tolerance to environmental conditions, Crassostrea gigas has become the oyster of choice for aquaculture in many regions of the world, such as Canada, USA, Mexico, France, Ireland, China and Japan [70]. Oysters can be collected from the environment (wild oysters) or cultivated to juvenile size (Figure 2) using wild or hatchery produced seed. In hatcheries, bacterial infections of larvae are common, and most frequently attributed to vibrios [71-73].

Thirty years after its introduction in France, mass mortalities have affected C. gigas threatening the sustainability of economically important aquaculture as well as biodiversity of wild populations. First, until 2008 oysters (juvenile and adults) were affected by what has been termed 'summer mortality syndrome' that occurred at a threshold temperature of $19^{\circ} \mathrm{C}$ and was geographically restricted [74]. This syndrome was influenced by physiological stress associated with maturation, abiotic conditions, genetic traits of the host, and aquaculture practices [74]. In addition, summer mortalities have been associated with the presence of several infectious agents: an ostreid herpes virus [75], designated OsHV-1, and bacteria of the genus Vibrio, namely $V$. aestuarianus, $V$. harveyi and strains belonging to the Splendidus super clade $[18,20,76]$.

Second, since 2008 deaths of juvenile oysters have increased considerably and current mortality levels range between $60 \%$ and $90 \%$ [77]. These events are more widespread because they occur at a lower threshold temperature $\left(16^{\circ} \mathrm{C}\right)$ and extend to all coastal regions (Atlantic, Channel, and Mediterranean) [78]. Previous research efforts have mainly focused on the hypothesis that this disease results from the emergence of a new genotype of the herpes virus, OsHV-1 $\mu$ var [78]. However, using SPF oysters, it has been observed that rapid colonization by diverse vibrios precedes the viral replication in filed conditions [7]. It has also been demonstrated that the virus is neither essential nor sufficient to cause juvenile deaths, whereas bacteria are necessary for the disease process [7]. This emphasizes the need to investigate the role of bacteria, particularly vibrios, in this disease.

Third, since 2012, the number of reported cases of adult mortalities associated with the presence of $V$. aestuarianus has increased considerably [79]. Interestingly, during the 20082012 period, this bacterial species had been rarely isolated from moribund oysters, suggesting the possible (re)emergence of $V$. aestuarianus as an oyster pathogen. 
Finally to date, these recurring mass mortalities have not been observed in other European regions. Invasive populations of $C$. gigas in Northern Europe have only been affected by isolated disease events (e.g. Norway, 2014 and Southern Wadden sea, [48]) or have been spared entirely, so far (e.g. Northern Wadden Sea). 


\section{Box 2. Population Genomic Tools to Understand the Functional Unit of Pathogenesis}

- Investigating the functional unit of pathogenesis requires an unbiased sampling strategy, which takes the ecology of the putative pathogen population into account rather than just collecting strains from moribund animals. Because vibrios are pathogens that can occupy a diversity, non-host associated habitats (Figure 1), analysis of animal-associated vibrios should be performed in the context of a metapopulation framework, i.e., by considering how dispersal connects hosts and alternate habitats, which may act as reservoirs of pathogenic populations (e.g., organic particles in the water column and zooplankton).

- Sampling should also reflect the biogeography of the host both in terms of genetic structure $[67,80]$ as well as disease history. The independent and genetically distinct sources of $C$. gigas in Northern Europe that represent a gradient of disease severity (Box 1) offer an excellent background for setting up such informative comparisons.

- The analysis of microbial population structure requires sufficient genomic coverage that can detect small differences with high precision since ecological populations are frequently differentiated below the commonly used 16S rRNA marker genes. Hence multilocus sequence analysis (MLSA) [81] or genomic sequences should be used to differentiate pathogenic populations from benign relatives. High-resolution genetic analysis can also be coupled with tools, such as AdaptML previously used to delineate ecological populations [29] that can link genetic structure to environmental features or habitats. This ultimately allows mapping of pathogenicity onto population structure to identify the functional unit of pathogenesis.

- Evolutionary analysis can highlight new virulence factors and mechanisms. Performing whole genome comparative analyses of closely related isolates with contrasting phenotypes from the same locality allows identification of genetic variation specific to pathogenesis and/or ecological niches. These evolutionary changes can be found on two levels: the core and the flexible genome. The exploration of allelic polymorphism within the core genome may show (i) an increased allelic diversity as a potential sign of balancing selection (i.e., selectively maintained polymorphism), or (ii) reduced allelic diversity indicating a gene-specific selective sweep. Different gene contents of the flexible genome, on the other hand, highlight genes possibly under frequency dependent selection [32] and/or genes under positive selection that have very recently been acquired but have not yet swept, or 
genes that are currently being lost from the population. Such analysis has obvious implications for pathogenesis determinant genes since these can be either part of the core or flexible genome.

- Functional manipulation of candidate genes (knock-out/knock-in) is essential for the formal demonstration of the predicted function. Several tools and methods have been developed to delete genetic elements [17] or remove plasmids [82] in non-model vibrios. The comparison of wild type and mutant strains in experimental infections can reveal the role of these genes in pathogenicity and/or host specificity. Mutagenesis efficiency can differ dramatically between closely related strains [46] and we strongly recommend testing the feasibility of genetic approaches prior to genome sequencing.

- Pacific oysters represent an up and coming model system in research leading to the development of a wealth of genomic resources including linkage maps, transcriptomes and a whole genome sequence [83-86]. Disease resistance encompasses a substantial genetic component that is responsive to selection [87] and the molecular diversity of oyster immune effectors is well characterized [88-90]. Such potential target genes can be manipulated by RNA interference [86] thus offering population genomic resources to functionally characterize host-pathogen interactions. 